\title{
Critical Care Nurses Perception towards Caring for Terminal Ill Patients and Ethical Dilemmas Faced, in a Tertiary Teaching Hospita, A Case of Muhimbili National Hospital in Tanzania
}

\author{
Article by Trustworthy Sithembile Majuta \\ RN-MSN, Texila American University, Tanzania \\ E-mail: trustworthymajuta@yahoo.com
}

\begin{abstract}
The purpose of this study was to assess the perception and to examine the ethical dilemma faced by critical care providers in a tertiary teaching hospital in Tanzania when caring for terminal patients. The study was guided by four specific objectives including, first to assess how the critical care Nurses perceive and understands end of life issues encountered during caring for terminal patients, second to explore the ethical dilemmas faced by Nurses during the course of duty in caring for terminal ill client, third to find out the psychological impact of end of life issues to critical care providers working in the ICU and lastly to determine the challenges encountered by critical care providers working in the ICU. The study was conducted from Muhimbili National Hospital (MNH) in Dar es Salaam, Tanzania. The population of the study was subgroup of nurses based on qualifications, expertise and experience, working from medical ICU, surgical ICU, cardiac ICU and CCU. All the nurses who met the above criteria were included in the study. The researcher applied both structure and unstructured interview to collect data. The study results shows that, majority of nurses come across end of life issues more frequently in ICU and most of them find these issues very uncomfortable. Nurses have negative perception towards a number of end of life issues for example they don't support the medical team to decide on withdrawing treatments. The other study result shows that nurses are psychologically and physically affected by end of life issues whereby most of them suffer from moral distress. Also, ethical dilemmas encountered by nurses in the ICU are mostly on truth telling; withdraw of treatment to vegetative patients and allocation of resources. Lastly the researcher found that, lack of adequate ICU training, lack of policies and protocol on withdraw of treatments and inadequate of equipments as the main challenges among nurses working in the ICU. The researcher suggest that the management must ensure well staffing as per universal standards, ideally nurse patient ratio in ICU should be 1:1, the procurement department must ensure the availability of equipment and consumables which all the nurses cited as one of the biggest hindrance to quality care provision and contributes to their dilemmas and stress. The hospital should do enough capacity building to cater for the size of the hospital, ideally and universal standards says, at least $10 \%$ of the total hospital bed capacity should be able to be accommodated into the ICU, hence Muhimbili hospital with a bed capacity of 1500 beds, must have at least 150 ICU beds.
\end{abstract}

\begin{tabular}{lrl} 
Abbreviation and acronyms \\
ICU & \multicolumn{1}{l}{ Intensive Care Unit } \\
MNH & $:$ & Muhimbili National Hospital \\
HCP & $:$ & Health Care Providers \\
DNR & $:$ & Do Not Resuscitate \\
DNAR & $:$ & Do Not Attempt Resuscitation Orders \\
CPR & $:$ & Cardiopulmonary Resuscitation \\
OJT & $:$ & On job training. \\
NM & $:$ & Nurse Midwife \\
BSc & $:$ & Bachelor of Science in nursing \\
MSN & $:$ & Masters of science in nursing \\
TAU & $:$ & Texila American University.
\end{tabular}


Texila International Journal of Nursing

Volume 2, Issue 2, Dec 2016

\section{SON : School of nursing \\ MUHAS : Muhimbili University of Allied Health and Technology \\ Introduction}

This chapter presents the background information, statement of the problem, research objectives governing this study which have been classified into two groups; general and specific objectives and the research questions of the study. Generally the research aimed to assess the ethical dilemma faced by critical care providers in a tertiary teaching hospital in Tanzania when caring for terminal patients. A case of Muhimbili National Hospital, in Dar es Salaam, Tanzania

\section{Background of the study}

The principles of critical care nursing are, To save life, To prevent complications, And To promote healing, hence the Nurses needs to be vigilant, scrutinizes, quick decision making and swift action to achieve the above for the fragile lives entrusted to them. ICU Nurses perceive critical care nursing as tiresome and stressful due to its complexity.

An intensive care unit (ICU) is a specialized unit, where facilities for critically ill patients are concentrated and the level of care is complex, intense and frequently life sustaining (Size, Borgstein \& Haisma, 2005). Ideally, units require one nurse per patient ratio to provide highly specialized care (Whiteley, Bodenham \& Bellamy, 2004).

Critical illness or injury usually results in an emergency admission to ICU. This may be physically and emotionally stressful to families who usually accompany their sick relatives during hospitalization (Williams, 2005). And also for Nurses. The admission happens without any opportunity for preparation, both emotionally and physically, for family members of critically ill patients.

This causes fear of death, uncertain outcomes, emotional turmoil, financial concerns, role changes, disruption of routines, and unfamiliar hospital environments (Medina, 2005). Critically ill patients are often incapable of making decisions regarding their own care.

This forces family members to make decisions on behalf of these patients, compounding the family members’ stressful experiences (Wasser, Pasquale, Matchett \& Bryan, 2001).

Patients admitted to ICUs require technical support for multiple organ failure, airway problems, and/or drug infusions to treat their severe conditions (Size, Borgstein \& Haisma, 2005). In addition to technical support provided to patients, nurses must address the psychosocial challenges and ethical conflicts associated with caring for critically ill patients and their families. More often Nurses are left with dilemmas when it comes to decision making and trying to up holds the ethics of autonomy, who to listen to, the client or the family? Ethically the patient has the right to choose what's best for self, but is powerlessness when one is brought to hospital in a critical condition.

Ethical dilemmas are the main common challenges faced by nurses, during practice almost on daily basis. These dilemmas can erupt amongst nurses themselves, or nurses and doctors and the worst between nurses and patients.

Conflicting obligations arises when the nurse is supposed to make ethical decisions between what to be done versus what not to be done, making it difficult to choose between the two. There are several factors that brings about ethical dilemmas such as, increase in consumer demands, shortage of resources, truth telling, withholding information, withdrawing therapy (end of life issues), and allocation of scarce resources, who to give first? And advance technology application (life support system, CCRT, Dialysis etc)

The environment of an intensive care unit has been recognized as a very stressful, high-tech, fast paced and emotionally charged atmosphere.

According to Prompahakul, Nilmanat, and Kongsuwan (2011) who conducted a study relating to nurses' caring behaviors for dying patients, it was noted that the most important aspect of nursing is caring and this is even more so in an intensive care unit (ICU). Apart from providing vigilant care to their patients and interacting on a daily basis with distraught families, critical care nurses confront death and dying, end-of-life decisions and ethical dilemmas very frequently. Furthermore, conflicting 
values, moral and ethical codes of conduct which exist amongst the multidisciplinary health team; namely, doctors, nurses, physicians and medical technicians, can create additional tensions in the critical care unit. In general, the very nature of nursing is stressful and a nurse's attitude about death and dying can be extremely complex to understand.

Critically ill patients usually stays for longer periods of time in ICU, and this allows the nurse to bond with the patient and the family. Patients enter the acute care setting in physiologic crisis and treatment needs to be aggressive in order for it to be lifesaving. Interventions are sometimes effective in treating and stabilizing these ill patients but it is estimated that as many as one in five patients dies in critical care. Critical care deaths represent the majority of hospital deaths and for many nurses, the loss of a patient can create intense feelings of grief, while for others, the reminder of their own personal tragedies creates a very trying and stressful situation Carlson (2009). Nurses suffering from such stress may lose their capacity to perform well in their jobs and this can result in job dissatisfaction and burnout.

According to a study that was conducted by Klein and Alexander (2003), it was found that medical personnel have a twofold responsibility in understanding grief, dying and death reactions. One is to help the bereaved to develop their own ways of coping and the other is to ensure that their own difficulties, needs and attitudes relating to death and dying of their patients do not compromise their psychological well-being. It was noted by the same author that the psychological impact and after events prevalent in a critical care nurses' working environment remains relatively unexplored. Michell (2010) also felt that repetitive exposure to resuscitative measures, end-of-life care needs, prolonging life by pharmacological and mechanical means and the continuous adjustment of these critical care nurses to this hostile environment, results in psychological disorders such as post-traumatic stress disorder.

Research articles have shown that there is a mounting trend towards withdrawal of treatment in intensive care units. Physicians are responsible for decisions concerning medical treatment, but as critical care nurses, it is within their scope of practice to carry out physicians' decisions. Sometimes the consequences of end-of-life pain, suffering and dying can be quite detrimental to the nurse. Medical technology has created new ethical dilemmas for medical professionals. Technological advances have allowed critical care medical and nursing interventions to heal impaired body functions. Technology also allows the medical profession to prolong life, in the critical care unit, often at great cost, both financially and emotionally, to both the dying and the living.

Even with such advanced medical technology worldwide, studies show, that there is a need for protocols, guidelines, and support services to be instituted to help the critical care nurse as she experiences ethical dilemma to make decision in the ICU (Carlson, 2009).

\section{Statement of the problem}

Ethical dilemmas result in feelings of discomfort, uneasiness which can further complicate to powerlessness, anger and frustrations. Ethical dilemmas are common with End of life issues which is a very sensitive subject and have proved to be a difficult challenge to both nurses and Doctors working in the critical care units, and have been cited as number one cause of stress and stressors in these units globally. Working in ICU can be traumatic for nursing personnel. Critical care nurses are faced with the repeated exposure to death and dying. Often they are involved in caring for patients who have a terminal illness, are actively dying or those who are faced with the possibility of impending death. Critical care nurses often have a difficult time coping with the stress that comes with caring for those who are dying. The nurse is affected more than other HCP because of the longest time frame spend with the patients and often burdened with lots of questions from the patients significant others about prognosis and decisions pertaining the delicate subject of life and death issues leaving the poor Nurse in a both ethical and moral dilemma on which is the best possible solution. Being an ICU nurse is a job with high demands, including the provision of family support and care. The unique demands and pressures of this role, creates psychological effect to nurses. Nurses suffering from such stress may lose their capacity to perform well in their jobs and this can result in job dissatisfaction and burnout. 
Texila International Journal of Nursing

Volume 2, Issue 2, Dec 2016

\section{General objective}

The general objective of this study was to assess the perception and to examine the ethical dilemma faced by critical care providers in a tertiary teaching hospital in Tanzania when caring for terminal patients.

\section{Specific objectives}

i) To assess how the critical care Nurses perceive and understands end of life issues encountered during caring for terminal patients.

ii) To explore the ethical dilemmas faced by Nurses during the course of duty in caring for terminal ill client.

iii) To find out the psychological impact of end of life issues to critical care providers working in the ICU.

iv)To determine the challenges encountered by critical care providers working in the ICU.

\section{Research questions}

i) What are the experiences encountered by critical care providers working in the ICU caring for terminal ill patients?

ii) What are the common ethical dilemmas faced by Nurses when caring for terminal ill patients.

iii) How and to what extent the end of life issues affects the psychological well-being of critical care providers?

iv)What are the challenges faced by critical care Nurses during practice caring for terminal ill patients.

\section{Significance of the study}

The completion of the study came up with useful information that helps the policy makers and political will, of the need to have a law or statutes that guide the HCP when they are faced with ethical dilemma and to be protected as well, when they are required to make decisions. Furthermore, understanding critical care nurses' ethical dilemma issues helps the health care delivery system prepare and educate these nurses more fully on issues relating to the needs of terminally ill patient as well as how to deal effectively with the stress that these situations cause. The study helped the Nurse to understand the legal rights aspect of the patient and prevents unnecessary conflicts.

\section{Limitation of the study}

There is no research undertaken without problems or limitations, in this study the researcher expected to come across a number of limitations, which in one way or another could prevent the researcher to perform the duty, though these limitations did not affect the validity and reliability of the study. The researcher expected that, as per Tanzanians culture and personal beliefs it would be very difficult for participants to discuss freely about for example options of withdrawing life support system. It is a very sensitive subject with many people preferring not to talk about it, including other senior medical personnel.

The researcher further expected to face resistance from respondent to participate in the interview because of being busy and some needing incentive. In order to work out these limitations, the researcher provided the respondents with adequate information regarding the significant of this study.

\section{Literature review}

\section{Introduction}

This chapter presented the literature review and the theoretical foundation of the study, definition of key terms and concepts, end of life issues in general, empirical studies and conceptual framework. The literature was reviewed from journals, reference books, working papers and research reports. 


\section{Theoretical review}

\section{Definition of key terms}

Ethics is our concern for good behavior. We feel an obligation to consider not only our own personal well-being, but also that of other human being." (Albert Schweitzer in Certo \& Certo, 2009).

An ethical dilemma is a situation involving conflicting moral claims and give rise to such questions as "what ought I to do?" and "what is the right thing to do?” And what harm and benefit result from this decision or action? (Davis et al, 1997). Ethical dilemma usually has no perfect solution and those making solution may find themselves in the position of having to defend their decision, (Ellis and Hartley 2004) The common dilemmas faced by ICU nurses are related to which is the best choice to make, should the treatment continue or not, who should get the first priority to life saving equipment such as ventilators? Want to take action beyond authority, tell or not to tell the truth, being patient advocacy versus maintaining relationship with other health team. All these dilemmas bring about feelings of confusion, discomfort, uncertainty and powerlessness to the nurse resulting in moral distress which has a very negative impact on patient care and outcomes as proved by Wilkinson study.

Intensive care is the specialized care of patients whose conditions are life threatening and require constant monitoring (Medterms, 2010).

Nursing challenges are emotional, intellectual and technical difficulties that ICU nurses face when caring for critically ill patients and their families since the ICU environment is different from hospitals' general wards (Farnell \& Dawson, 2006).

\section{Advance directives}

Advance directives are a written statement, made in advance by an adult (ie, 18 years of age or older), that appoints someone to make healthcare decisions if a person becomes unable to make decisions and unable to communicate his or her wishes, and/or provides instructions that describe the kind of care a person would want or not want under particular conditions. Physicians, other members of the healthcare team, and family members use these documents to ensure that a person's preferences are honored, (Christine and Teresa, 2005).

Advance directives do not need to be written or signed by an attorney. Most states require 2 witnesses to the document; many states mandate that the witnesses cannot be the person's relatives, heirs, or healthcare provider. A person can change his or her advance directives at any time or even revoke the document completely. The change or revocation may be made in any way that the person is able to communicate. A third party cannot complete, change, or revoke the directive of another.

\section{Types of advance directives}

The 2 major types of advance directives are the Living Will and a Medical Power of Attorney. Both documents are executed only if the person lacks decision-making capacity and is unable to make personal treatment decisions. In some states, a person can give directions about organ and tissue donation within the context of these documents.

\section{- Living will}

A Living Will is a document that expresses wishes about medical treatment at the end of life. It takes effect only in situations of terminal illness or permanent unconsciousness. Some states have a statutory Living Will document. For example, the state of Iowa created a "Declaration Relating to the Use of Life-Sustaining Procedures." All states except 3 (Massachusetts, Michigan, and New York) have statues that provide for Living Wills, (American Bar Association Commission on Law and Aging, 2004)

\section{- Medical power of attorney}

A Medical Power of Attorney also referred to in some states as "Durable Power of Attorney for Healthcare” or "Patient Advocate Designation," differs from a Living Will in 2 important ways: 
Texila International Journal of Nursing

Volume 2, Issue 2, Dec 2016

- The Medical Power of Attorney allows a person to name a partner, also called an advocate, proxy, or surrogate, who legally expresses the patient's wishes about medical treatment when the patient is incapacitated.

- The Medical Power of Attorney becomes activated in any situation in which a person is unable to make decisions whether the circumstances are terminal or not.

All states except Alabama have statutes that recognize some form of healthcare proxy. Most address all healthcare decisions, but some are limited to terminal conditions, (American Bar Association Commission on Law and Aging, 2004)

\section{Use of advance directives}

To date, despite the demand for autonomous decision making by patients, few Americans have prepared advance directives. Although no recent nationally representative studies exist about completion of advance directives, data from previous population-specific investigations suggest that fewer than 25\% of Americans have advance directives, (Gilllick M. 2004).

There are many reasons for this phenomenon, including the following:

- $\quad$ Lack of knowledge about the document and its use,

- $\quad$ Perceived complexity of the process and documents,

- Fear that care will be limited without knowledge and/or consent,

- $\quad$ Denial of morbidity and mortality, and

- $\quad$ Spiritual and cultural values and beliefs

Most people assume that families and healthcare providers will make the "right" decisions for them if they lose their decision-making capacity. However, studies show that families and providers are not able to accurately predict patients’ decisions, (Covinsky DI, Fuller JD, Yaffe K, et al, 2000)

\section{Barriers}

Healthcare providers can play a central role in assisting individuals to complete advance directives. Unfortunately, many providers lack basic knowledge about the documents, have little training on how to counsel individuals about preparing advance directives, and may not appreciate the value of the documents in practice. Some providers believe that advance directives are not necessary for young, healthy patients. However, cases such as Nancy Cruzan and Michael Martin, which provided the impetus for advance directives legislation, show that death does not discriminate.

Facilitating discussions about advance directives takes time that may not be directly compensated, and lack of compensation for providing the service has been identified as a barrier. In addition, because physicians do not accept death as an appropriate outcome of care, they are reluctant to discuss a plan of care that would not be medically aggressive, (Reisfield G, Wilson G, 2004)

\section{Values and religion}

When discussing advance directives, patients must explore their individual values. Values are core beliefs regarding what is right and fair in terms of our actions and our interactions with others, and what individuals believe to be of worth and importance in their lives, (Navaran F.2004). The patient's values will guide him or her in making healthcare choices. The following 3 types of values should be considered in this situation:

- Personal values, which form the basis for behavior and include both moral and non-moral beliefs

- Cultural values, which are indigenous to a culture or people versus an individual

- Professional values, which are derived from the individual's professional group

Healthcare professionals' responsibility is to create an open environment where patients are allowed to educate us about their individual values. Values emerge in conversation as part of the creative process, and thereby patients can explore their basic life values, quality-of-life values, and healthcare and medical values.

Health care providers belong to different religion with different perception when it comes to such issues moral values. The Islam (Qur-an) teaches acceptance of life, not rejection nor withdrawal. "Nor take life- which Allah has made sacred except for just cause. And if anyone is slain wrongfully, we 
have given his heir authority (to demand Qisas or forgive) but should not exceed bounds in the matter of taking life he is helped"

\section{End-of-life decision-making}

End-of-life care decisions are challenging, because emotions and ethics are attached to actions that can lead to the hastening or perceived hastening of death, (Lippincott W\& Wilkins; 2000.)There are fairly universal legal and ethical prohibitions for certain actions, such as active euthanasia, which is the direct killing of a person.

However, other actions, such as physician-assisted suicide, are now legal in Oregon and Washington. In addition, there are other actions that are legally and ethically permissible, but are considered morally objectionable by certain individuals or groups, often centered in faith and/or geographic communities.

These actions include withdrawal of nutrition and hydration, withdrawal of ventilator support, and use of sedation for extreme pain and other symptom control (known as palliative sedation, previously called terminal sedation), (Lorenz KA, Lynn J, Dy SM, et al,2008)

What makes end-of-life decision-making even more challenging for the provider, especially during discussions with patients and surrogates, is that the end-of-life terminology that is often used, (such as allowing to die, euthanasia, assisted suicide, physician-assisted death, etc.) can have different meanings to different people. Providers need to be clear in their use of terminology, ensuring that the patient or surrogate is using the term in a manner similar to the provider, (Lippincott W\& Wilkins; 2000.)

Health care providers must be sensitive to, and respectful of, the diversity of moral beliefs surrounding end-of-life care, and must clearly understand the moral justifications for actions that may be perceived as hastening death. For example, the "principle of double effect" is often invoked to justify the use of high doses of narcotics to effectively treat pain in terminal illness (although hospice advocates would argue that it is irrelevant here) and the use of sedation to treat intractable symptoms, (Quill TE. 2000). Most doctors and nurses who work in end-of-life care know that, although the narcotic is given to treat increasingly unbearable pain, drugs like morphine given in increasingly high amounts that produce unconsciousness can also hasten death.

Health care providers must also be aware that while legally and ethically, there is no difference between withholding therapies in the first place and withdrawing therapy once it has been started, family members may feel differently. While a clinician's moral obligation is to his or her patient during end-of-life care, the patient-family unit is more often the object of care, even when other family members are not actually patients. Clinicians may have moral duties to the family unit as well as to the patient, especially when end-of-life-care choices run counter to prevailing community sentiments.

\section{Do not resuscitate/do not attempt resuscitation orders (DNR/DNAR)}

Choosing to forgo resuscitation is the most common end-of-life care decision. This decision, based on patient or surrogate consent, is enacted when a clinician issues a Do Not Attempt Resuscitation (DNAR) order, which instructs medical personnel not to begin cardiopulmonary resuscitation (CPR) when a patient suffers a cardiac or respiratory arrest, (Lippincott Williams \& Wilkins; 2000.)

At one time CPR was seen as a medical obligation, rather than a therapy that could be withheld at a patient or surrogate's request. As statistics of survival to hospital discharge after CPR have accrued, it has become apparent that survival rates after CPR are dismal for most people with end-stage diseases, and that resuscitation offers little or no benefit to the terminally ill; in many cases it simply prolongs their suffering. This dilemma has sparked discussions among ethicists about futility and CPR, (Cantor et al 2003)specifically about whether there is a need for informed consent before initiating a DNAR order in certain terminally ill patients, (Lynn J, 2001).

Currently, patient or surrogate consent is generally required before a DNAR is issued. The issue of how to handle a situation in which the patient or surrogate insists on CPR despite the fact that it would 
Texila International Journal of Nursing

Volume 2, Issue 2, Dec 2016

be medically futile is an area of intense debate, (Lippincott Williams \& Wilkins; 2000.)At least two states have enacted statues that address this situation, (Cantor et al 2003).

While withholding resuscitation for the dying makes clinical sense to those in the medical profession, patients and family members may see these decisions as an attempt to limit other therapies or restrict care. DNAR discussions between provider and patient are, in fact, often the logical starting point for broader discussions about therapy limitation(s); since a DNAR order, by itself, only limits one specific therapy, cardiopulmonary resuscitation.

It is important that patients, their surrogates and hospital staff all understand the limited nature of a DNAR order and appreciate that it is consistent with both aggressive disease-fighting care as well as comfort measures only. By emphasizing the limited nature of DNAR and sensitively exploring patient goals for care, clinicians can help their patients understand and navigate other potential treatment decisions that might arise. Providers can also uncover and address any inconsistencies in choices; for example, patients who request resuscitation, but simultaneously refuse treatment for the underlying condition that will likely result in cardiopulmonary arrest, (Cantor et al 2003). Even after a decision is made, the clinician and patient can discuss the issue of resuscitation again when a related therapy decision needs to be made.

DNAR discussions with patients and families should always involve honest and sympathetic dialogue, with the health care provider trying to determine as clearly as possible what are the patient's or surrogate's wishes. To further emphasize the value of such discussions, one study noted that in almost one in three cases, the patient's preferences not to use CPR were different than the physician's perception of what the patient wanted (Teno JM, 1995). The study reinforces the importance of DNAR discussions and other end-of-life issues with patients or surrogates.

\section{Cultural issues in critical care nursing in the ICU}

According to (Urden, Stacy and Lough, 2010) the critical care nurse needs to 'know the patient' in order to individualize and humanize nursing care. Beliefs about health and illness are deeply rooted in a person's culture.

The response of a patient or family member to the diagnosis or treatment may be strongly influenced by his or her values and culture. Nursing literature acknowledges the importance of providing culturally sensitive patient care, however there is little evidence of cultural awareness in the critical care nurse's daily assessments and interactions with patients and families. According to Lee, Anderson and Hill (2006), sometimes culture plays a vital part in end-of- life decision-making. An individual sometimes cannot make an independent decision because the family as a whole is considered to be the smallest decision-making unit. Trans-cultural nursing refers to a formal area of study and practice that focuses

On providing care that is compatible with the cultural beliefs, values, and lifestyles of individuals. A cultural assessment includes the patient's usual response to illness as well as his or her cultural norms, beliefs, and world views (Hudak et al., 2008). They highly recommended that critical care nurses must recognize their own individual biases and examine their personal values and beliefs about health and nursing care. Many health beliefs and values are based on commonly held European and American values that have influenced nursing. Some of these beliefs are; beliefs in individualism, informed consent, orientation toward clock time, and belief in God as being the most powerful being. There are other shared beliefs, such as the belief that modern technology and scientific measures will improve a person's overall ill-health. The influence of such values on other cultures may negatively impact on nursing care (Hudak et al., 2008).

\section{Moral and ethical obligations in critical care nursing in the ICU}

In critical care, the appropriate use of technology and information in patient care can be useful yet very crucial. The ICU is run on complicated technology and is a place where critical decisions about life and health are made. Patients in the ICU are acutely ill and although nurses and other health care providers make moral choices and are faced constantly with ethical dilemmas in everyday practice, choices are sometimes difficult and create feelings of uncertainty, conflict, or distress (Zomorodi and 
Lyn, 2010). ICU nurses view their role as consisting of multiple goals, with patient care being only one aspect of their daily routine.

The same study showed that the nurses' role in the ICU is currently focused on the curative model, and nurses feel ill-prepared to focus on a palliative, patient-centered role.

In today's society, however, it was found that death is no longer an everyday thought and is difficult to predict and this has resulted in frustration about the nurses' role at the time of death.

Ethics is an integral part of the foundation of nursing. According to Searle (2000), ethics refers to the study of those standards and values that helps a critical care nurse answer questions about what is right or good. Morals are personal or codes of conduct that define the critical care nurses expected behavior. Not only are they the standards of behavior and values to which critical care nurses are committed as members of society and of a practicing profession, but ethics and moral obligations characterize a critical care nursing profession (Hudack et al. 2008). A study done by Klein and Alexander (2003) regarding issues that cause moral distress to ICU nurses states that anything that interferes with a nurse's ability to provide optimal patient care has the potential to create moral distress in an ICU. The writer argues that issues such as difficulty acting according to rules and regulations, lack of support structures, rendering of poor quality care, incompetent colleagues and lack of medical resources such as medical equipment are as some of the moral and ethical conflicts facing an ICU nurse. In another study done by Langley and Schmollgruber (2006), it was noted that at the end-of-life, the patient, the family, doctors and nurses are the primary persons involved in the actual ethical decision-making process and they are the human face of care. Decision-making is frequently a frustrating process which can sometimes be disadvantageous to the patient.

\section{Death and dying in critical care units and its impact on critical care providers}

In a study done by Wingate and Wiegand (2008), it was reported that most deaths in a hospital, occur in a critical care unit and that American views on death and dying are very much influenced by Western medicine. It was noted in this study that the Western culture of medicine is diverse and constantly evolving. Critical care medicine and nursing tend to view topics like disease, health, death and dying in a broader context.

Statistics reflected in this study show that most deaths in the United States occur in the critical care settings of hospitals. However, Western medicine remains based on scientific, rational and objective principles.

The American Association of Critical Care Nurses has maintained that critical illnesses should never become an ordinary experience for nurses (Morton et al., 2005). These authors argue that knowledge and care go hand in hand: one without the other does not translate into quality care. Therefore, the specialty of critical care requires high levels of preparation and in-depth knowledge to enable the critical care nurse to cope with death and dying. When patients and families hear diagnoses and end results, it can be devastating if the critical care nurse has limited or no knowledge in how to deal with the patient and his or her significant others.

The nurse and patient can feel lost, helpless, and powerless and may handle grief poorly and with fear. Wingate and Wiegand (2008) also stated that even though Advanced Directives are used in America to assist the families of critically ill patients in making decisions regarding the patient's death and dying or in treatment decisions, most patients admitted to critical care do not have Advanced Directives.

According to Niederriter (2009), surveys done by the Canadian Nurses Association (CNA) revealed that the essential value of providing compassionate and appropriate care to all individuals facing a life-threatening illness should always be upheld and that quality end-of-life care for a dying patient can be best provided if there is collaboration amongst the multidisciplinary team. CNA also suggests that nurses are probably one of the most important role-players in an ICU because they assist and provide direction to the dying patient. Nurses were part of a complex process that was a physically, psychologically, emotionally and a spiritually intimate one which allowed the nurse to develop a therapeutic relationship with the dying patient. 
Texila International Journal of Nursing

Volume 2, Issue 2, Dec 2016

Research studies that were conducted in Eastern and Middle Eastern countries reveal a different perspective on dying. For example in India, studies discussing issues relating to coping and dealing with terminally ill patients noted that death and dying was an emotive and sensitive issue that could not be talked about casually by health care workers.

There is no cultural acceptance of withdrawing life support or treatment. However, the spiritual tradition of the Indian subcontinent glorifies and welcomes a dignified acceptance of death. It has also been found in this study that since Hindus believe in reincarnation and trans-migration of the soul, they tend to sometimes approach death without fear (Mani, 2003). Another study done in Saudi Arabia surveyed people of the Islamic faith and the way death was perceived by them. According to this study, there were no standardized nursing plans or strategies that helped nurses care for a terminally ill or dying patient. It was however; very important that nurses acknowledged and respected Islamic religious beliefs and rituals to make the dying process smooth. It was also noted in this study that Islam prohibits assisted suicide, mercy killing and euthanasia. The issue of organ donation is still under debate and is surrounded by a lot of religious controversy (Salman and Zoucha, 2010).

A Swedish study done by Fridh, Forsberg and Bergbom (2007) reported that there was a significant role that the family played in making death and dying decisions for the patient in an ICU.

It was also noted that the critical care nurse's knowledge of the Swedish culture of death and dying and family presence in an ICU at the time of death was seen as effective end-of-life care. In countries such as Belgium, end-of-life decisions are made every day (Vincent, 2006). What was noteworthy in this study was that Belgium practices euthanasia and very often in their ICUs, life supporting treatment is withdrawn or withheld prior to death in the critically ill patient.

The study does make note that controversy still surrounds this legislation as doctors and nurses working in an ICU are faced with many moral and ethical obligations of their practice, as many feel that their role function is to ensure well-being and maintain life rather than to assist in ending it.

Dying and death has a different connotation for people of Africa. Many Africans believe that the spirit of the deceased remains in the world and that the dead person can come back embodied in another person. It is believed by many Africans that death stands between the world of human beings and the world of spirits (Searle, Human and Mogotlane, 2009).

In a study done by Eyetsemitan (2002) on the cultural views of death and dying in Nigeria, it was found that the loved one had time to prepare both emotionally and cognitively for death before it occurred. The author suggested that this made coping after a death much easier for significant others to handle. Similar to other African perspectives on death, this study also found that religious beliefs and personality characteristics were important aspects that helped the individual cope with the dying or death of a loved one.

\section{Communication issues in a critical care unit}

Good communication in an ICU is a matter of insight and self-awareness, not just language skills (Langley and Schmollgruber, 2006). There will always be inherent difficulties with the ICU environment because of its unpredictability and lack of quality time for critical care nurses to care for dying patients and the patients' families. Other problems include communication between doctors, nurses, and patient's families in an ICU. It is said that end-of-life care or care of the dying can be improved if communication measures ensure that all members of the multidisciplinary team are working towards the same goals for the patients and their families (Fouche, 2006).

According to Urden, Stacy and Lough (2010) the critical care nurse needs to 'know the patient' in order to individualize, humanize and communicate nursing care. Life experiences and value systems can influence the way a critical care nurse perceives death and dying. Events such as the death of a family member or friend, religious upbringing, spirituality, near death experiences and personal attitudes can determine nurses' thought processes related to grief, death and dying.

Experience with death or caring for the dying is emotionally draining. In a study by Dracup and Bryan-Brown (2005), it was argued that developing communication skills amongst the different staff in an ICU was just as important as developing other clinical skills. It was also noted that 
communication between the multidisciplinary team and the dying patient can be tiresome and daunting.

Critical care nurses become frustrated and have reported that they are uncertain if their role is to postpone death or extend life. The same study highlighted end-of-life communication has being a specialized type of therapeutic communication that requires specialized education. Having communication skills and family centered communication, are seen as subjective approaches in medical science, and are considered key components of implementing end-of -life care in an ICU (Curtis 2001a).

A study carried out by Elliot and McKinley (2008) suggested that even though patients in critical care units are too ill to participate in decision making, significant others should be involved in patient care and treatment. According to the same study, critical care nurses can assist by providing physical and emotional comfort to both the patient and the relatives. According to Dracup and Bryan-Brown (2005), communication amongst the health care team, the patient and the family is essential to devising an adequate care plan for the patient. Patients' responses must be reviewed and communicated each day.

The authors of this article also stated that intensive communication, between healthcare providers and families in an ICU, led to either continued support for patients with the potential to survive or allowed for early withdrawal of life support if it was deemed ineffective. It should be noted that frequent and respectful communication among caregivers about short and long term goals regarding death and dying issues, will not only help patients and families adjust their expectations about what is able to be achieved by ICU care, but will also make difficult decisions and discussions less difficult.

Zomorodi and Lyn (2010) stated that although the traditional role of the ICU nurse was high technology curative care, the increased incidence of disease, death and dying amongst populations has made it clear that ICU nurses felt ill-equipped or unable to communicate with regards to handling end-of-life or palliative care. Critical care nurses have reported not being adequately equipped to communicate with the dying patient or the grieving relative. Nurses often appeared to express a sense of helplessness, frustration, uselessness and guilt when working with patients and families in death and dying situations. Some nurses also appeared to draw from their own life experiences when relating to dying patients or grieving relatives, whilst others became so distressed by the situation that they shied away from any discussion on the topic.

In the critical care unit, death is no longer treated as a natural outcome of life but rather seen as a medical failure. Often the critical care staff is so focused on saving a life that they ignore that the quality of the life saved and the pain and suffering inflicted to save the life are ignored. The findings of the study that was done by Zomorodi and Lyn (2010) revealed that nurses lacked preparedness when dealing with end-of-life care in a critical care environment. Prolonged exposure to the stress and trauma that accompanies patients death and dying, can compromise the critical care nurse's ability to cope, both at home and at work. The study also suggested that emotionally disturbing experiences with death led to ICU nurses presenting with hyperactivity, aggressive outbursts, sleep disturbances and impaired concentration which led to withdrawal, emotional instability and pessimism.

\section{Psychosocial issues in a critical care unit}

People facing death tend to suffer from an inability to find meaning. In this last chapter of their lives, lots of issues affect these critically ill patients, ranging from: the inability to be able to deal with family and loved ones, a total dependence on others, a loss of capabilities, the change from being sole breadwinner into a burden on others, being unable to perform the daily activities of living, being cast out of the world of the living, guilt, a sense of abandonment; and from anger about all of this (Hardwig, 2000).

It was noted in a study done by Klein and Alexander (2003) that health care professionals are not impervious to the emotional impact of the demise of those under their care and even though most nurses are taught Kubler-Ross's grieving process, some of them even go through this process (namely denial anger, bargaining, depression and acceptance) when working with dying patients (Hudak et al. 2008). For the critical care nurse, negotiating these steps of grief and coping with the psychosocial 
Texila International Journal of Nursing

Volume 2, Issue 2, Dec 2016

demands of the dying patient and their loved ones may be experienced as being more stressful than the complex medical management of patients. One-third of ICU nurses experience burn-out syndrome and this was especially true when the goals of the health care team were at odds with critically ill patients and their families (Niederriter, 2009).

Findings from a study done by Glaser and Strauss (2004) suggested that the relationship between the nurse and family members of the dying patient is an important factor in a nurse's ability to cope. This study revealed that the coping abilities of nurses were positively influenced by those family members who understood and responded to the needs of the dying patient and adhered to ICU policy.

The authors further argued that it is within this profound, reciprocal, emotional, and interdependent relationship between nurses and relatives that the nurse caring for the dying patient or one who has died can continue to provide care despite their experiences of overwhelming emotional and physical strain. The ICU can be an unnatural and threatening environment for the patient, and their families as well as the nurse. As much as each one has their own role to play, they are all dependent on each other. The need to finalize or part with emotionally complex information to the relatives or patient, can be very emotionally taxing on the critical care nurse. Information such as the poor progress, poor clinical outcomes, or poor prognosis of a patient can lead to vital and important decision-making.

According to Brysiewicz (2006), family members are often unable to comprehend the loss of their loved one and that unexpected deaths can compound the impact of grief on family members. The same study revealed that family members also needed to be included and involved in the decision making process and care of their loved one. Hudak et al. (2002) stated that there are still gaps between nurses and families of the dying patient. With modern technology and the state of the art care provided by an intensive care unit, critically ill patients can now be kept indefinitely alive. However, emotionally overwhelmed and confused families can create great distress in even the most committed and dedicated health care worker. In this study it was further revealed that the greatest challenge to humane and appropriate care for critically ill patients is related to the psychosocial adjustment of the family. One needs to understand that, being confronted with the possibility of death is an emotional experience and not a technological one and is an experience that will have a life altering implication for the family concerned. The authors also stated that the emotional features of an ICU, namely the symptoms evoked by ICU stays are just as threatening, as the physical elements such as, life support machines and the sound of alarms beeping. These tend to create a sense of vulnerability because of the emotional and physical dependency of the patient in ICU.

\section{Empirical studies}

According to Gondwe, Bhengu and Bultemeier (2011) Nurses experienced numerous challenges in meeting the perceived needs of ICU patients' family members. The main challenge was a lack of preparedness.

Other challenges included a lack of adequate ICU training, and lack of policies to manage family members. Nurses also reported inadequate preparation for providing sustained information to family members; managing the continued presence of family members; promoting family members' abilities to rest; and for dealing with specific religious practices and competing demands for patient and family care.

Lack of experience, the absence of written policies and inadequate education and training of ICU nurses were identified as major barriers for providing support to family members. Farnell and Dawson (2006) indicated that less experienced and less well trained nurses need a period of socialization in order to acquire knowledge and skills to care for patients, before the nurses can provide support to patients' family members. Through experience, nurses learn to know their patients and these patients' family members (Pryzby, 2005). The nurses' knowledge of dealing with families in crisis affected their attitudes toward family members of critically ill patients. Working in an ICU is emotionally and intellectually challenging as the environment is different from that of the general hospital wards (Farnell \& Dawson, 2006). In the study, none of the nurses had formal ICU training.

Proper family support requires adequate staffing. Nurses were reportedly usually in short supply in the ICUs and could not adequately meet family members' needs in addition to caring for critically ill 
patients. Engström (2006) reported that ICU nurses wished that one nurse could take care of family members, while another one cared for the critically ill patient, especially when they had just arrived in the ICU. In this study, having one nurse look after the patient and another one take care of family members was unfeasible due to the shortage of nurses.

The continuous presence of family members in the ICUs was stressful to the ICU nurses, interfering with the care rendered to the ICU patients. This "perceived interference" might be due to different cultural, educational and religious backgrounds and dynamics, which could affect family members' behavior in ICU.

Engström (2006) reported that families from different cultures were problematic due to diverse views of appropriate behavior when patients are critically ill. It was reported that nurses needed to be culturally sensitive to family members' needs and should integrate preferred cultural routines into patients' nursing care plans.

Naidoo (2011) conducted the study to explore the critical care nurse's experiences of death and dying. The findings of this study revealed that issues such as communication, multicultural diversity, education and coping mechanisms relating to caring for the critically ill and dying patient are essential in nursing education and practice.

Critical care nurses need to have support networks in place, not only to assist in providing care, but also for their own emotional support.

\section{Conceptual framework}

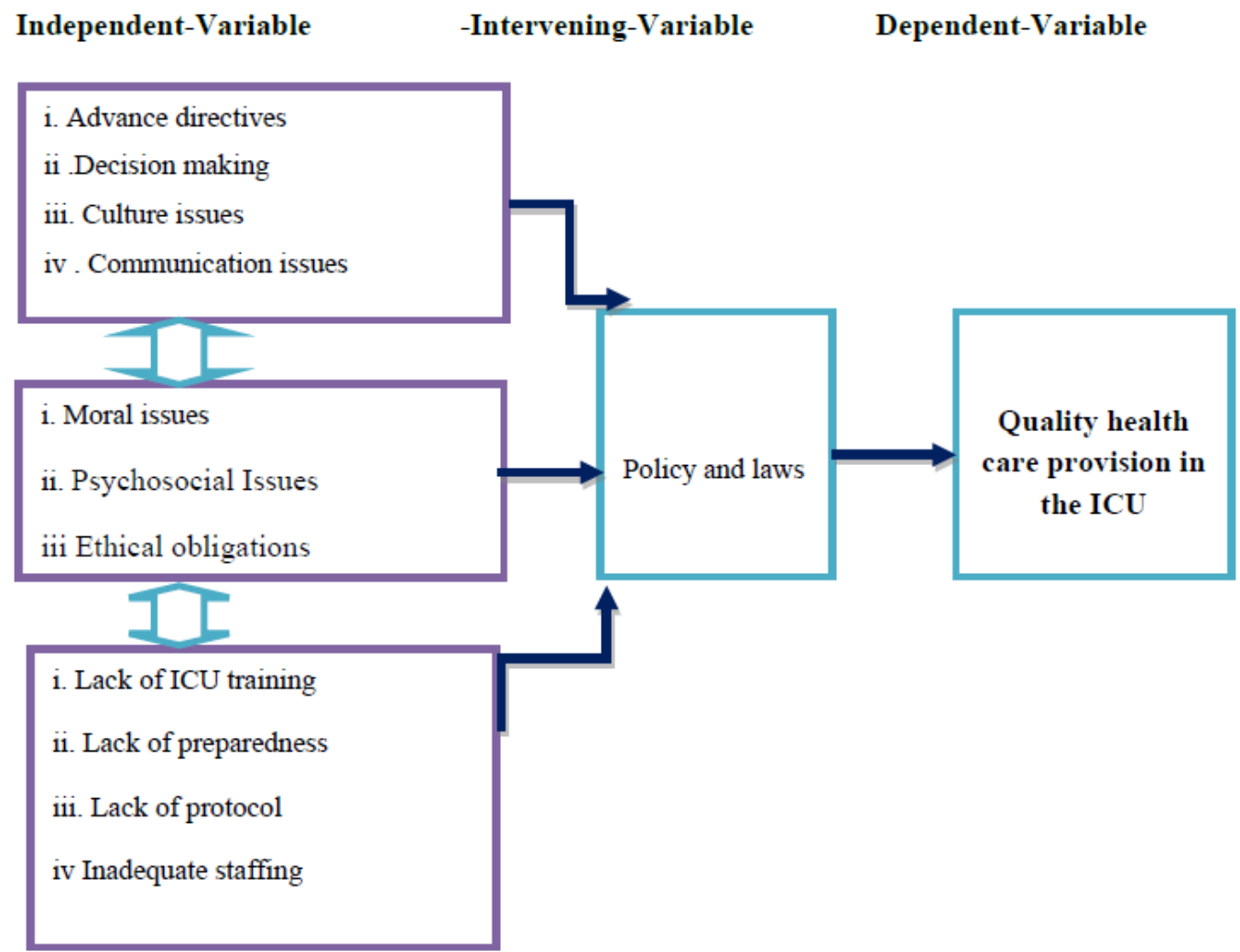

\section{Intervening variables}

In this study, the policy and the law/statutes has greater influence on facilitating the quality of health care services offered by nurses in the ICU to critically ill patients. This is because the policy guides the whole process of decision making that nurses are required to make, while the law is the one which protects critical care providers when conflicts arise especially from family members and other activist. 
Texila International Journal of Nursing

Volume 2, Issue 2, Dec 2016

\section{Dependent variable}

Quality care provision is treated as dependent variables and it is the main focus of the research, and can be influenced by other factors mentioned in the independent and intervening variables.

\section{Relationship between variable}

The study describes how independent variables; that are, advance directives, decision making, culture issues, ethical obligations, communication issues, psychosocial issues and moral issues together with challenges encountered by critical care providers such as lack of ICU training, lack of preparedness, inadequate staffing, lack of resources and lack of protocol determines the extent of ethical dilemma to critical care providers. This indicates that good performance of one, or more of the independent variables listed above have direct impacts on the quality of care given by nurses.

\section{Methodology}

\section{Introduction}

This chapter basically provided an explanation on how the study should be conducted. It described the area of study, population, research design, sample size, sampling techniques, data collection methods and tools, data processing and analysis for this study.

\section{Area of study}

The study was conducted from Muhimbili National Hospital (MNH) in Dar es Salaam, Tanzania. The area was selected due to the availability of large number of nurses working in about three different ICU available in the hospital. MNH is the referral national hospital and receives a large number of patients who are in a terminal condition. The area was further chosen because the researcher was familiar with the environment as she works from the same hospital and therefore expected high response rate from the respondents.

\section{Research design}

Research design is a plan of how the research will systematically collect and analyze the data needed to answer the research questions. Thus, it is a generation of evidence that is suited both to a certain set of criteria and to the research question in which the investigation is interested (Bryman, 2001). Kothari, (2004) adds that research design constitutes the blue print for the collection, measurement and analysis of data. According to Polit and Beck (2008: 17) qualitative research is a type of scientific research and investigation that seeks to understand a given research problem or topic from the perspectives of the local population it involves. Qualitative research is especially effective in obtaining culturally specific information about the values, opinions, behaviors, and social contexts of particular populations. The strength of qualitative research is its ability to provide descriptions of how people experience a given research issue and it provides information about the 'human' side of an issue. Qualitative methods are also effective in identifying intangible factors such as social norms, socioeconomic status, gender roles, ethnicity, and religion. This type of data can be acquired by indepth interviews

In order to explore the experiences of the nurses with end of life issues and ethical dilemma they face while caring for terminal patients, a qualitative study using a descriptive phenomenological approach was used to guide the study. This type of approach is useful when the focus is on describing the subjective experiences of the participants and in examining the beliefs and cultures experiences of the subjects. A phenomenon is a fact or occurrence that appears or is perceived. A phenomenological paradigm is concerned with understanding human behavior from the participant's own frame of reference (Polit and Hungler, 2004: 246). 


\section{Sample size and sampling techniques}

\section{Population}

A population is a totality of objects under investigations, Kamuzora and Adam 2008. In this study, the population of the study was subgroup of nurses based on qualifications, expertise and experience, working from medical ICU, surgical ICU, cardiac ICU and CCU. All the nurses who met the above criteria were included in the study.

\section{Sample size}

The Researcher used the judgment or purposeful sampling technique to select a sub group of nurses basing on the qualifications, expertise and experience. All nurses who had specialized in critical care nursing, and all those who had a vast experience of above five years working continuously in the critical care area and are well with ICU routines, protocols and operating standards procedures were included into the study, as shown from the table3.1 below

Table 3.1. Distribution of Sample Size

\begin{tabular}{|l|l|l|l|}
\hline Descriptions/subjects & Population & $\begin{array}{l}\text { ICU } \\
\text { trained }\end{array}$ & $\begin{array}{l}\text { Over 5yrs, } \\
\text { experience }\end{array}$ \\
\hline Medical ICU & 22 & 2 & 6 \\
\hline Surgical ICU & 21 & 1 & 5 \\
\hline Cardiac ICU & 18 & 1 & 6 \\
\hline CCU & 11 & 1 & 3 \\
\hline Total & 72 & $\mathbf{5}$ & $\mathbf{2 0}$ \\
\cline { 2 - 4 } & \multicolumn{2}{l}{} \\
\cline { 2 - 4 } & Total sample size 25 &
\end{tabular}

Source: Created by the researcher 2016

\section{Sampling techniques}

According to Martin N Marshall, judgment or purposeful sampling technique in qualitative studies is more intellectual strategy as compared to others, more so considering the researcher vast practical knowledge and experience of working with critical patients, contributed in the selection of the technique. Sample is the number of items selected from the universe to represent a population study. (Adam and Kamuzora 2008). The sample size of this study would be 25 respondents, drawn from medical ICU, surgical ICU, cardiac ICU, and CCU as indicated in the above table created by the researcher.

\section{Data collection methods}

In order to obtain the relevant data and information concerning the topic under the study, the researcher employed various methods of data collections. Both primary and secondary data were collected.

\section{Primary data}

This is data observed or collected directly from firsthand experience, and not yet manipulated by any other person. Such data is provided by the subject in the study sample through scheduled interaction using structured/unstructured and probing question in form of interviews method. Kothari, 2004.

\section{Interview}

This is a data collection method which was used to collect data and all other required information from the respondents. Both structured and unstructured interview questions were asked to the study respondents. 
The in-depth interview was conducted with the use of an interview guide containing a demographic section as well as a central question to focus the discussion. The purpose of interviewing was to understand the essences, meaning, and values, that participants attributed to the phenomena under study. Interviews were scheduled for thirty minutes, for each participant. The interview was carried out to all 25 nurses who participated in the study.

\section{Secondary data}

A secondary data involved the gathering or use of existing data for purposes other than those for which they was originally collected. These secondary data may be obtained from many sources, including literature, industry surveys, compilations from computerized databases and information systems, and computerized or mathematical models of environmental processes (Kothari, 2004). The secondary data was collected through documentary review available from the ICU are explained hereunder:

\section{Documentary review}

This is another method used for the purpose of collecting the secondary data to supplement the primary data. The information collected by other researchers. The researcher went through various documents within the ICUs which contains the relevant information about the study. For example, minutes of family meetings, protocols, guidelines, and policy and information posters.

\section{Data processing and analysis}

The researcher analyzed the collected information by using qualitative (descriptive) method. The analysis was based on the statement of the problem, research objectives and research questions. Qualitative analysis involved categorization of data from interviews and field notes into common themes. Interview responses were coded and analyzed by pen and paper, thereafter, contents were transcribed.

\section{Data analysis, observations and findings Introduction}

This chapter is about analysis, interpretation and discussion of the findings. A detailed discussion of the results is done based on research objectives and questions and similar findings are cited wherever required. The chapter will also serve as a baseline for making conclusions and recommendations. Data is presented and discussed with the help of simple figures as a means of summarizing and clarifying on the matter discussed for each finding with regard to the study objectives.

\section{Demographic characteristics of respondents}

This part presents the demographic characteristics of informants involved in the study; it presents sub topics such as, gender of respondents, age of respondents, type of orientation in the ICU' and ICU work experience. 


\section{Gender of respondents}

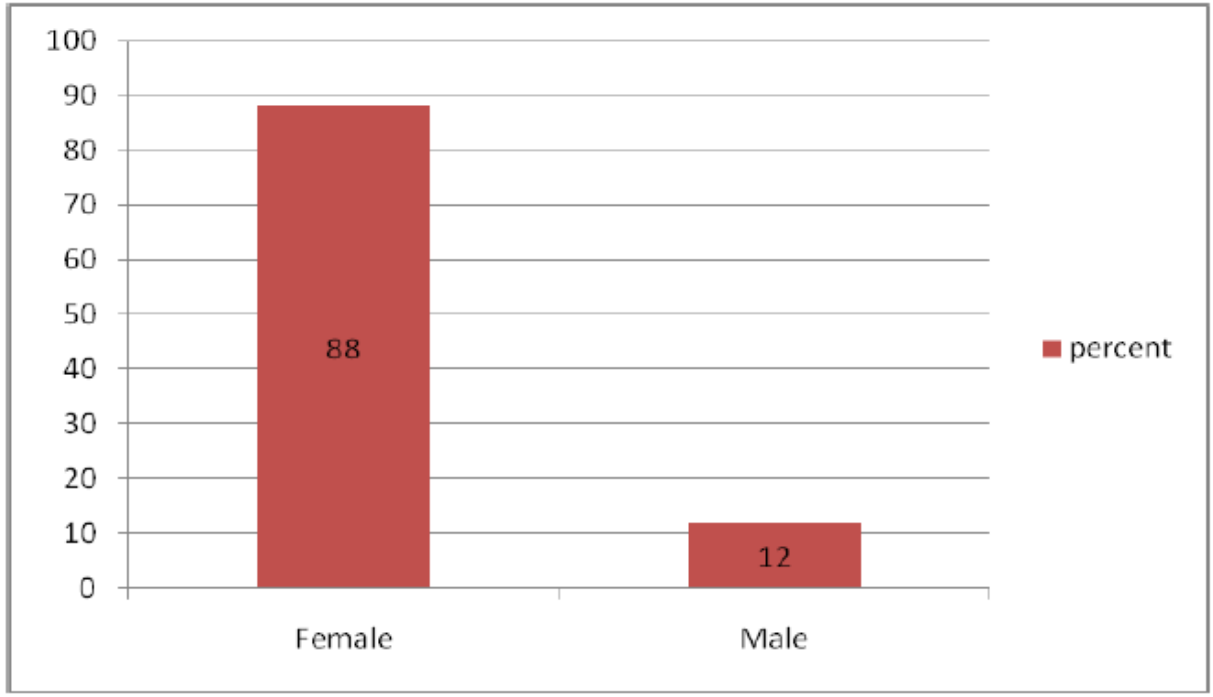

Figure 4.1. Gender of respondents

Sources: research findings 2016

In this study sex category of respondents were analyzed in order to make the research gender sensitive. The study shows that of all respondents who participated in this study through interviews, (88\%) were female whereby (12\%) were male. This shows that, the main part of this statistics were collected from female than male. The variation was due to availability of respondents whereby females were more available than men. This can be probably due to the nature of the job in which most nursing positions are occupied by female. For that case therefore, it worthy to conclude that the research was not gender balanced.

\section{Age of respondents}



Figure 4.2. Age of respondents

Sources: research findings 2016 
Regarding the age group of respondents, the study found that, majority of participants who participated in this study were in the age group ranging $41-50$, this group constituted $49 \%$, being followed by the other age group of 31-40 which constituted $28 \%$. It was further found that, $13 \%$ of informants were in the age group of nurses with more than 50 years, few were found in the age group of 20-30 (10\%). These findings implies that, most participants who participate in this study were active adult who have worked for a good number of years with the organization expected to give a valid information about the motivation packages in their organization and its impact to the organizational performance.

\section{Working experience}

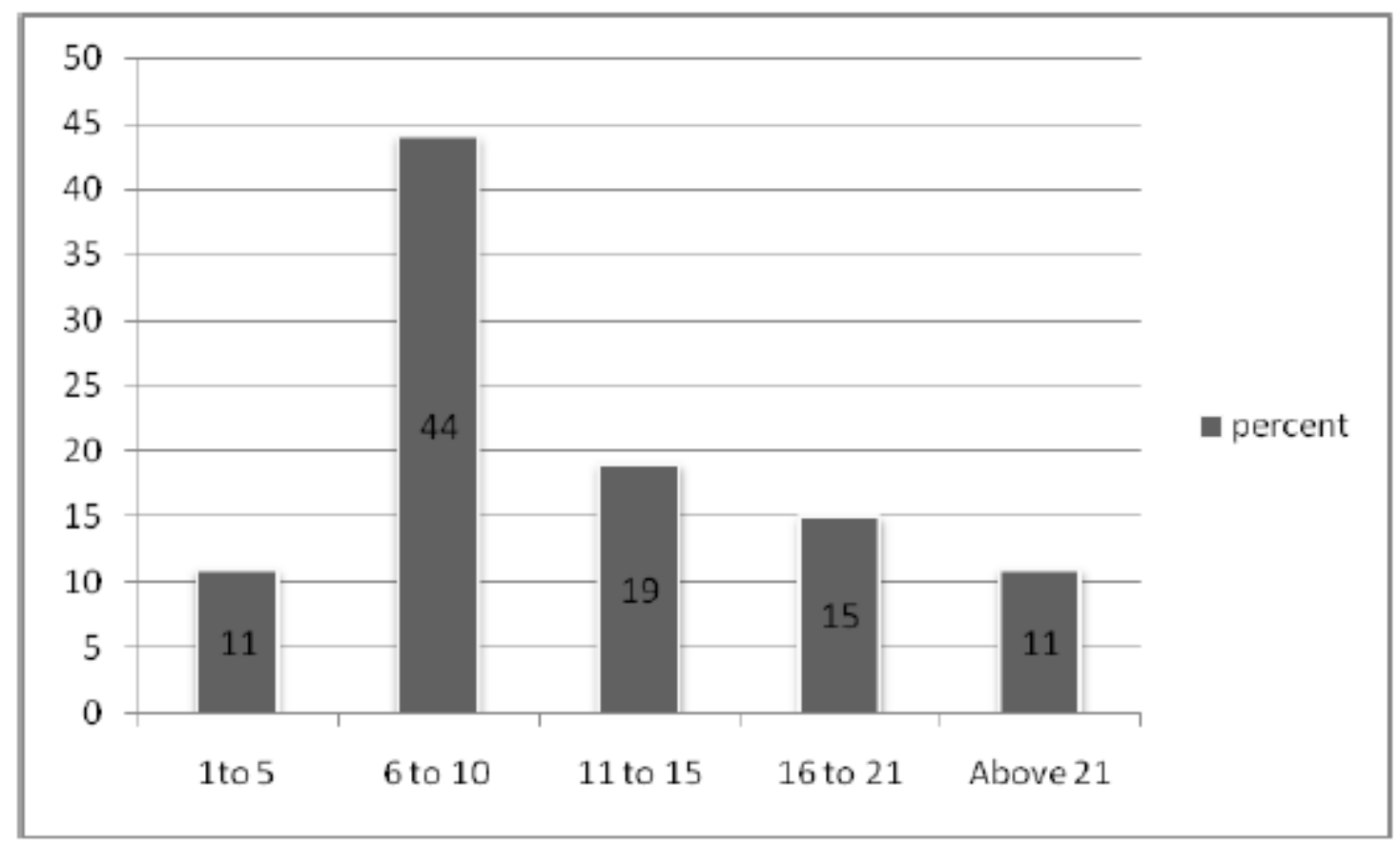

Figure 4.3. Work experience

Sources: research findings 2016

Working experience is the major factors to determine the ability of the respondents and the correctness of the data provided by them. This is also a big concerned in this study, hence respondents were interviewed on their experience. Experience was grouped into five groups. Research findings show that grand majority of nurses approached have been working with the ICU for more than five years (6-10) this constituted $44 \%$. Followed by (19\%) engaged for 11 to 15 years. This shows that the majority of the respondents had enough experience to support the reality of their responses on motivational packages and its impact to the organizational performance. 




Figure 4.4. ICU specialized VS non specialized nurses

The figure above shows number of nurses with specialized training ICU viz -a - viz those who work based on experience. Out of a total number of 72 nurses working in the ICU, less than $10 \%$ are formally trained in critical care.

\section{Assessing how the critical care Nurses perceive and understands end of life issues}

The purpose of this research objective was to assess the perception and understanding of nurses working in the ICU towards the end of life issues. Issues discussed in this objective includes, decision making, advance directives, information dissemination, nurses and family relationship and standard operating procedures.

\section{Awareness of nurses on end of life issues}

The report shows that, majority of nurses understands and come across end of life issues more frequently in ICU basically and most of them find these issues very uncomfortable and have negative perception towards a number of end of life issues for example they don't support the medical team to decide on withdrawing treatments, they would advocate for normal dearth to occur unless it is the patient himself who determined his/her fate by choosing what should be done as far as DNR is concerned or withdraw of the treatments, religiously and culturally nurses don't support that, they have a strong belief that God himself is the only one who can determine ones dearth despite the prognosis or brain dearth.

There are so many incidents on end of life issues especially for patients who develops brain dearth especially if it is a child, it is difficult to make a decision on one's life. Nurses believe that only God can determine when to take one's life. In the ICU environment there are patients even noting while on the machines, and it is discouraging for the nurses to care for a brain dearth client whereby they know nothing will come out of it, somehow it is a waste of resources which would have benefited the other patients whose prognosis are good. And since ICU have limited number of ICU beds factually by continuing nursing that patient one will be blocking a bed for others, the dilemma comes that, who are we to withdraw treatments, and culturally this is a very sensitive issue to even discuss.

"We critically think that, if the patient will not benefit from the admission, for instance, brain dearth patient, you can't change anything, the patient will die, let him die peacefully and I advocate normal dearth to occur. We must not block other patients who can benefit from the ICU admission by admitting patient whom we already know they can not benefit from the ICU care".

"When it comes to vegetative cases, I will opt to allow normal dearth to occur because I feel and think nobody has the right to take the life, it is only God who can out someone's life, and even culturally it is a taboo, unheard off". 
Texila International Journal of Nursing

Volume 2, Issue 2, Dec 2016

"I feel the patient with terminal illness should be left to die in peace, no termination of life support system for we are not God, it is only God who can terminate one's life and also culturally it is not acceptable, so I will advocate for one to be given a chance to live until God says it is enough".

"I think if a patient is terminal and vegetative there is no need of prolonging life especially if there are no way of reviving that individually, why wasting space, resources that can benefit others. I personally feel that aggressive measures should not be done especially to brain dearth patients but allow normal dearth to occur while preventing suffering by taking care of the pain palliative and ensuring comfort”.

It was stressed that, when the patient becomes vegetative nurses and doctors thinks on the best way forward in the best interest of both patient and the family, weighing the pros and cons raising questions such as is the patient going to benefit from this management? Is the condition reversible? What is the prognosis? If all these answers are not for the benefit of the patient, then withdraw of active management is inevitable.

\section{Standards operating procedures}

The researcher was keen to find out if there are standard operating procedures which guides the operations of nurses in the ICU, research findings shows that a large number of nurses are not aware and have not seen such manual, but few especially those who have worked with the ICU for longer period hardly said that, there are established standards though most nurses have no idea because they don't practice it. She demanded that all nurses should practice the laid down standards as far as patients care is concerned. The challenge on the implementation of the laid down procedures comes due to the fact that most nurses do not have special skills and competences to follow it. Furthermore, standard operating procedures depends mostly on the availability of resources if everything is available such as drugs, consumables and equipment then standard operating procedures will be followed smoothly, but now it is not possible to do so because there is a lot of improvisation in the ICU set up hence one cannot do procedures properly due to the said reasons.

With regard to the standard of care given to critically ill patients, informants said that

"I can't say it is $100 \%$, but we are giving the best to all our patients, depending with the staffing. We wish to deliver, however situations like staffing shortage, lack of consumable also specialists sometimes not there and you wish they would be around to take care or to discuss the way forward of the patient".

It was further said that as the time goes the quality of the service is improving, compared to the time when they started working in ICU, they have a feeling that they are moving toward standards.

"As an ICU specialist I think I deliver up to standard care, years and years of repeatedly caring for these sick patients have made me a very good nurse and improved my working standards as they say practice is the best teacher".

\section{Nurses, patients and family relationship}

Maintaining the relationship between nurses, patients and families is crucial in the ICU setting because the family depends much on the nurses who are like bridges between them and the medical team.

Due to severity of the patients conditions most relatives are scared and stressed and it is the nurse who need make sure they understand what is going on reassuring them and encouraging them to actively participate in the patient care in a way they could do such as visitation, bringing food and toiletries and anything that might be needed during the stay in the hospital as well as supporting the patient psychological. Nurses said that, they have a good relation with relatives because there are visiting times for the family and most of the families ask how their patients are progressing despite the seriousness of the problem. However, family members expect to hear good news only, but there are situations that one need to tell the exact situation and this brings about problems when they hear what they would not have expected.

There are several challenges which arise for example, sometime family members have asked other people and have a different information and when a nurse give them something else it brings 
uncertainties. Another challenge comes on payment of hospital bills. Charges for general wards and ICU are different, but many relatives lacks that knowledge, the first contact health providers are not giving fully information concerning payments creating misunderstanding leaving the nurses in a difficult position to talk about the bills. Conflicts occurs because people are different because of the previous situations that they are or have been experiencing, with some having nursed their patient for a long time or that particular patient was the bread winner, or financial status not good, with all the money finished on hospital bills.

The nature of relationship that nurses have with family members is more supportive and they are working together and assessing what is the needs, what is the family expectations and together they talk and plan on the way forward. Nurses also involve the family in patient RX for example if the patient stays in the ICU for a time and needs a tracheotomy nurses seek consent from the family.

"We had a baby who had been on ETT for more than 2/52 and the parents were informed of the need for tracheotomy and the further was scared so the mother and further discussed the issue and agreed we also discussed with them and explained the benefit".

Also nurses always ask relatives to bring all the consumable used for the patient treatment that is not available such as central line, TPN. Some families agree positively but some don't agree, they feel once they bring the patient to the hospital and pay charges the hospital is obliged to pay for everything. Many of the ICU patients have low economic status so this difficult them to afford. Also the political people tells the public that hospital is free and want them to believe there is free treatment from all government hospitals which lead into public not trusting the nurses at times thinking that nurses are stealing their things.

The study found that, nurses always try to create good interpersonal relationship with the family, however not all relatives are good; some are arrogant and always conflicting with nurses. Nurses involve them in patient management when they ask them to contribute in things like bringing food, toiletries and any other consumable, sometimes, some relatives stop coming to the hospital after being asked to contribute due to poor economic status until when nurses have to look for them, some would have been nursing for a long time until they are broke and lose hope.

Respondents further said that, generally there is no problem as far as nurse patient, family interaction, the only problem comes when the patient and their family are not given enough information, however if you give enough information concerning the illness and care most of them understands, so we constantly health advocate them and answer their questions. My relation with patients is very good, most of the ICU critically ill patients are unable to speak due to critical adjuncts connected to them, so nurses need to use their communication skills to obtain necessary information and give it to the patient.

Conflicts arise due to misunderstanding because the families always have selective hearing, they want to hear good news only and expect miracles from nurses, once they find patient not improving they always have the nurse to blame and when things are good credit is given to the doctor for a job well done. Some relatives appreciate that nurses are taking care of their patients but the problem comes on cost sharing issue, people expect to receive free treatments, so the nurse, client relationship is affected by the issue of payment, with many thinking that it is the nurses who want the public to pay not the government, so enough education is important to educate the mass the significance of cost sharing.

\section{Decision making}

With regards to decision making, the researcher was keen to find out if nurses are involved in decision making, research findings shows that, there are situations that a nurse can make decisions but only in emergencies situations, cold cases and long term plan as far as patient management is concerned, it is outside of their jurisdiction. Such decisions are only done as suggestion for the doctor to give the final decision, nurses advise doctors on the best possible management because they work as a team. It also depends with the altitude of the doctor in charge; some never take any suggestions from the nurse. They said that, ICU is somehow different from other places set up, nurses make lot of decisions as far as care and management is concerned. In a set up where there is no doctor coverage 
Texila International Journal of Nursing

Volume 2, Issue 2, Dec 2016

24 hours/7days a week an ICU nurse is forced to make quick decisions especially life serving but for cold cases, decisions are in consultation with all the team members and consultants as well as the family. Most of the time ICU nurses decide what benefits the patient even in the absence of the specialist or doctors or any other workers.

"We do make decisions on nursing issues and incase of emergence we decide treatments and interventions within scope of our practice otherwise most decisions are left to doctors since they have the upper hand on the patient".

The role of nurses in decision making is that they have to consider the patients' rights and stand for the patient, after predicting any problems they need to act and advice the doctor on the way forward and sometime even provide their own decision since they are the one with the patient most of the time. In times of emergence they can make their own decision to save the patient even giving medication and then discuss with the doctor later about my action.

"We sometimes make decisions as far as the patient's information of advice during ward and in cases of emergence we do make decisions to save life and all nursing issues we make decisions for example, a patient has high temperature I will not wait for the doctor, I have to administer antipyretic".

It was further stressed that, nurses are involved in decision making as part of the treatment team, they play their role of advising the doctor on what to do and sometimes in the absence of the doctor during emergencies when there is no time to call, the ICU nurse has to assist and save the patient though decisions are limited to nurse's scope of practice. Doctors always ask the nurses on the way forward since nurses are the ones with the patient longer time. In case of emergence or absence of the doctor nurses make decisions to save life. Even if it is not an emergence case one cannot wait the patient to deteriorate, especially those nurses who are ICU trained.

\section{Information dissemination}

The study findings shows that, nurse are only supposed to give information that pertain to nursing care and the supportive care family members are supposed to do, it is more of general, only information pertaining to the nursing scope of practice, anything deeper and outside should be referred to the responsible person. They also communicate information concerning consumables that may be needed by the hospital such as toiletries, food etc. Generally speaking the information given by nurses is very limited, the scope of practices allowed nurses to convey to the family.

The extent of information that nurses give to the family depends on what exactly they want to let them know for example, if they want to explain the patient condition, general condition, how the patient is doing? They always have to assess the perceptions of the relatives before and also ensure that they are capable of giving that information or otherwise they need to refer the issue to someone else, so it really depends with what type of information the relatives wants to know, when it is about the bill, they usually refer to the responsible person. Or if the family wants to see doctors for the medical diagnosis, the nurse will arrange for the meeting.

"As a nurse I always talk about the care that i render to my patient, the scope of our practice limit us a lot on information as we are not allowed to discuss in detail the patients diagnosis, only the doctors can do that".

Usually information given to patients by nurses, it is about the conditions, treatments and if there is anything that nurses would like the family to bring such as toiletries, special food etc., Information in detail about the disease is explained by the doctor who is managing the patient. Nurses are limited by the scope of practice as how much information to disseminate so most of the time we only discuss the nursing care only, disease process we always refer to the doctor for further explanation or reinforce what the doctor has said. Therefore, the information given by nurse is limited to those things within the nursing scope of practice but when it comes to prognosis and medically treatments; it is the doctor who should do that. 


\section{Opinion on DNR (do not resuscitate)}

Study findings show that, it is the doctors who give that order depending on the prognosis and diseases process. There are patients admitted and it is discovered that the patient has reacted the end of the stage, there is nothing to be done, in case of cardiac arrest then DNR is indicated and clearly documented in the patients file, and then it is the duty of the nurses to keep that patient comfortable, pain free and support till the end. Nurses keep on providing all the nursing care to allow normal dearth to occur.

Even though doctors make decisions based on the prognosis and disease process but as a nurse it is difficult to see patient gasping and not doing anything, there are times nurses have to overlook those decisions and continue to resuscitate patients against the doctors' order, it is one of the dilemma faced. Majority of nurses did not support withdraw of treatments completely; they suggested some assistance to be given to a patient such as oxygen. Nurses would allow normal dearth to occur, their personal belief does not support decision on one human ending life of another person. They believe it is only God who can decide that, furthermore the culture does not support as well. Nurses believe on trying, sometimes if they resuscitate the patient may come up. Below are more reaction of the nurses regarding the issue of DNR.

I don't support the DNR order, especially when the patient is now in the ICU, what is the point of bringing the patient into the ICU and later on say don't do anything, for me once the patient is brought to the ICU then resuscitation should be done. They should not admit patients who are not going to benefit from the ICU wasting resources.

We had one patient, who was very sick with Cancer larynx with metastasis, the patient started gasping and I asked the doctor why do we resuscitate, and he said DNR? I asked why he said the patient would not benefit from the CPR and let her RIP.

I think it is ok if the diagnosis and prognosis is poor and we clearly see the patient will not benefit from that then the patient should be allowed to normal dearth, however it must be made as comfortable as possible.

\section{Advanced directives}

With regard to the availability of advance directives, the study findings shows that, such a thing is not common in this society because of the culture and religious beliefs, though with the few that nurses have been exposed to for example, one isolated case whereby the patient told nurses that incase of cardiac arrest the patient was not willing to be resuscitated and his wishes were granted. Culturally it is considered as witchcraft to decide your fate such as end of life issues; people do not even want to discuss such issues for they make them uncomfortable.

In Africa there is no such kind of thing, one can stay with his grandma even up to 100 and above years and one still want to see her, and it is culture we can't say no. Also people are not knowledgeable, nurses think that even if African people would be educated on the issue still they would not accept it. It was further added that, African society lacks knowledge on advance procedures such as organ transplant, people believe that if one donates any organ will end up being used for rituals or scared of being buried without any organ such a thing will bring curse to the family.

Few nurses have come across patients with advance directives. One nurse told the researcher she came across with a patient who clearly indicated that in case of any incapacitation he should be left to die peacefully, it was a verbal order, fortunately it was honored.

"I have come across a patient with advance directives because of religious beliefs, his religion didn't allow blood transfusion (Jehovah witnesses) and the HB was 3 and going down, so at the end no transfusion was done and the patient died".

Therefore advance directives in the African society it is not common, least one will be labeled a witch. Probably there is a need to educate the society of such development and keep up with the others in the world. 
Texila International Journal of Nursing

Volume 2, Issue 2, Dec 2016

\section{Psychological impact of end of life issues to critical care providers in the ICU}

The purpose of this research objective was to find out the psychological and physical impact of end of life issues to critically care nurses working in the ICU. The study findings show that the majority of nurses are psychologically and physically affected by end of life issues as illustrated here under.

Study report shows that, working in the ICU have both psychological and physical impact to the well being of nurses. It affects nurses so much-because clients are patients they would have wished them to get well unfortunately they die- at times when nurses are trying to do everything possible using all their efforts and time hoping the patient to get well but at the end of the day the patient is not improving at well. This situation affects nurses very much. Sometimes the diagnosis alone is so serious and prognosis poor such that one wishes the patient to die and rest in peace.

Nurses said that, most of the patients in the ICU are critically ill and caring for those patients have improved their knowledge and skills in caring for them. The practice was mentioned to have both positive and negative impact on the nurse's life. The positive way is that it gives them much knowledge through experience that they get repeatedly. The negative way stress that comes seeing those critically ill patients affects them psychologically and socially. Caring for the critically ill patients consumes much of their time, preventing them to participate in social issues because when they get home they are very tired and cannot do other things.

"Time to take care of my family is now limited and even when am off had to reach and search some work related information to update myself on new information now and then".

A nurse specialist with critical care training and more than five years' experience nursing for critical care nurse said that, caring for the critically ill patients is stressful just seeing people suffering and approaching end stage. This affects nurses a lot more psychologically and may progress to physically due to stress and fatigue which may end up prone to sickness such as frequent cold, flue and sometimes abdominal upset and the worst which is burnout and prevent nurses from giving the best to my patients and their families. Some nurses decide to resign from work due to stress and burn out if left uncontrolled. She said that, stress in ICU has left nurses powerless; hopelessly with so many dilemmas on what were they supposed to do in order to change the situation. Also sometimes guilty conscious always hunt nurses, which leave them with questions like, did i do the right thing or not? All these leave nurses with mental torture and regrets.

Another nurse mentioned that, caring of critical ill patients is overwhelming, as she tend to put herself in their shoes and she feels exactly the same way they do, and this always reminds her that in this world human being are passing. This also reminds her that dearth as reality in life and makes her do all the best she could to help them with dignity by providing everything possible. The work is stressful, physically it is tiresome because of the workload that comes after caring of sick people, there is much to done leaving me exhausted and fatigued at the end of the day. She said. Even after going home she fails to carry out her duties as a mother and wife.

Embarrassment is another negative psychological impact among nurses working in the ICU, this was more stressed by a trained care nurse holder of master degree who previously used to work more with children, took care of children who were very ill in a situation where she found herself she has nothing to do to save their life and at the end of the day it is very embarrassing when they die. She noted:

"The situation affects me personally a lot, because I lose hope and always not happy seeing the very sick patients suffering and find myself unable to save their life, it is very embarrassing, and after work when I go home ' $m$ sad, and therefore my family is also affected when they see me unhappy they become sad as well".

She added that, socially her life is disturbed because she always think of those patients even when she is home, her mind would be wondering if the next day she would find her patients alive. She could always ask questions to herself like what she should do in order to save patients life; this situation disturbs the whole social interaction at home.

The study findings further shows that, nurses are also very much affected by the shortage and complexity of equipment necessary to provide best care to the critically ill patients. One respondent 
mentioned that at first her experience in critical care unit was in the emergence medicine and biggest challenge was equipment but when she was moved to ICU there was a big different as far as equipment was concerned despite that those two units are almost similar. She added that, dealing with highly technological machines was a big challenge, since she had no formal training in ICU, relying more on experiences and on the job training which she felt was not enough. Shortage of drugs and delay in supply of ordered medication affects her so much as a nurse, in as much as she would like to offer patients the best services, such delay always leaves her feeling helpless.

"I want to help patients but I have nothing to use and this frustrates me a lot. You look at the patient's condition deteriorating wishing you could do something such as HB but the blood bank people are telling me they have misplaced the form and they need or have misplaced the specimen now I have to start again while the patient is deteriorating".

On the other hand, nurses reported that, caring for the critically ill patients in the ICU improves their spiritual wellbeing, knowledge and skills as it has become part of their life. People admitted in the ICU are very ill some are nearer to die, the situation makes nurses get close to God because seeing a dying patient it is something that one don't get familiar with, so through working in the ICU nurses see themselves becoming close to God something which affects their life. The impact was also extends to one's family as one of the respondents noted that:

"When I see a patient with eclampsia and I go home and see my sister is pregnant, i ask myself is my relative going to be ok. Is the pregnant going to be well? It is kind of stress because i reflect what I have seen in the unit, in one way it is strengthening but in another way it is weakening me."

On psychological and physical effect, she said that:

Psychologically I have been affected especially when the patient dies and you have put all your effort to save. It is good when you are with a patient and patient prognosis is good gives a sense of good and people tend to appreciate more our work unlike the opposite. It is not easy to see a patient in pain and suffering, knowing very well the prognosis is poor and the patient is not getting better and there is nothing you can do to stop the suffering. Physically I just feel tired due to too demanding of caring for critically patients also when the mind is stressed the body tends to be fatigued as well."

\section{Exploring ethical dilemmas faced by nurses during the course of duty in the ICU}

The purpose of this research objective was to explore ethical dilemmas faced by nurses caring for the critically ill patients in the ICU. The study found that nurses come across a number of dilemmas such as truth telling vs confidentiality, withdraw of treatment vs equality, resource allocation vs equality and so forth.

\section{Ethics and ethical dilemmas in the ICU}

The researcher was eager to find out the awareness of nurses as far as the term ethics and ethical dilemma is concerned, findings shows that, majority of nurses understands the meaning of ethics and ethical dilemma in connection to their work; some definitions given by nurses are as follows:

"Ethics are things done within the legal action like guidelines. Ethical dilemma is where by two solutions conflict with each other, there is no right or wrong and the choice is difficult, for example a brain dead patient on mechanical ventilation decision to withdraw or to continue treatment is one of the common dilemmas."

"Ethics are guidelines which direct a nurse in nursing practices without violating the law. Ethical dilemma are issues whereby as a nurse am caught in between whether to do or not to do. Both solutions brings uncertainty, there is no best way of doing it. For example, a very sick patient who collapses and the doctors are saying prognosis is poor and you wish to resuscitate at the end you don't know how best to do, DNR or to proceed, what if the patient dies, so at the end of the day am left powerless and in a dilemma not knowing what to do."

"Ethics are guideline that guides us to care for the clients as per expected legal aspect of framework. Ethical dilemma these are challenging moments in nursing whereby a nurse is caught in between on what to do or what not to do, the nurse is left in confusion on the best possible solution and as nurse almost on daily basis we face such dilemmas in our practice, the dilemma can be among 
Texila International Journal of Nursing

Volume 2, Issue 2, Dec 2016

ourselves, patients or with family members. For example truth telling VS confidentiality, withdraw of treatment VS the right bill, resource allocation VS equality, all these leaves nurses uncertainty on what to do."

Findings shows that, nurses come across ethical dilemmas more often between ourselves and with patients, especially in resource allocation for example which one to assign to a ventilator, truth telling against the confidentiality such as HIV Status, whereby the affected patient does not want the family to know and the family are the one caring for that patient; they claim their right to be informed. Brain dearth patients, to switch off life support system or to allow normal dearth to occur, older patient and younger patient who to put first on the ventilator?

Costs telling is another ethical dilemma faced by nurses in the ICU, nurses said that, previously they used to tell relatives about payments but it turned back to them that they are giving relatives information that they are not supposed to give. With some admin telling them to give relatives that information, and relative perceive it like nurse are contradicting with doctors saying this and nurses saying that. For example when a dearth occurs and nurses failing to issue certification because of no payment the conflict arise, relatives blaming nurses for wanting money at the expense of their grief. Nurses have to be very careful and tactical in dealing with situations, encouraging relatives to bring whatever they have and arrange for the social worker to take care of the situation.

During the interview nurses shared with the researcher a number of cases encountered in the ICU that brought dilemma as presented here under.

A five years boy post adenoctomy and pre operatively patient well, intra-op patient got hypoxia! And the further was in denial, could not even see that the baby was vegetative and it was difficult to break the news, for him the baby was just sick and hoping to wake up one day, full of hopes. On the day news was broken to him nurses had to call one colleague who was close to the further and finally they were told the child was brain dead, it was very touching to the side of the family and the medical team, it took some times about two weeks for them to accept the news.

A girl with abortion did not want the parents to know, she was critically ill in a ventilator due to septic shock and the parents wanted to know the source of sepsis, what to do?. A client with very low HB but the religion says no to transfusion and the patient is dying. Two clients with one ventilator which one to choose? It was said that these dilemmas always leaves nurses with lots of questions, uncertainties on what is the best possible decision because any decision made has no right it conflict with the other.

\section{Determining the challenges encountered by critical care providers working in the ICU}

The purpose of this research objective was to determine challenges encountered by nurses working in the critical care unit and suggest possible measures to address the challenges. The study found that, lack of skills and competence among nurses, absence of the policy and protocol on withdraw of treatments, inadequate equipment and poor supportive suspensions from the management as the main challenges encountered by nurses in the ICU.

\section{Nurses' skills and competence}

Lack of skills and competence among nurses working in the ICU was said to be the major challenge in the ICU. Respondents said that only $25 \%$ of the nurses are competent and skilled enough to care for the critically ill patients either because of experience or by training. Most nurses in the ICU do not have special training to care for critically ill patients. The management system of rotating nurses out of ICU was said to have a very negative impact on having nurses who are competent and skilled, because it takes some time for a nurse to master skills of critically care and when one becomes competent she is moved to another unit totally different, making her lose all skills. Nurses should be given the opportunity to follow their area of interest and specialize along that and more formal training should be rendered.

Sometimes nurses working in the ICU are very new and are supposed to be trained how to care for critically ill patients and it takes time to learn because as people they differ, others catch up quickly and rapidly and others are slow learners. However there are few nurses who are very competent 
especially those having special trainings on caring for the critically ill patients. They are very competent, they have grasped well and are able to teach and help others but they are very few. I'm very competent because of the long period I have stayed in the ICU over these years due to repeatedly practicing and doing procedures, I find myself each day gaining experience and becoming more competent. There is need for more mentorship to new staffs and assessment of their competence, skills be done on regular basis and the training accordingly. Some nurses mentioned that, some senior nurses try to help those who are not competent but most are left unsatisfied with the answers to their concerns, emphasizing the need of formal training.

Most of the nurses' lacks practices and experiences since they are not critically care trained, there are very few nurses who are trained, only five to be precise out of about 30 nurses working in the ICU, and this means that there is a need of formal training and on the job training. They lack service training, self-esteem and interest, they further lack skills on the psycho-social part, the emotional side of the nursing, and therefore training is needed especially for those nurses who do not have ICU training as a specialist in order for a patient to be treated holistically.

At least $50 \%$ of the nurses should have the knowledge when you have more than 5 beds. I think more training to nurses should be done. Most of them have a general nursing and this needs special training, and on the job training which must be done locally.

For the moment care given to patients is not up to date due to lack of trainings in order to provide specialized care. The on the job training given now is not enough. Most of things are learnt from the exchange programs whereby some nurses from abroad visit the hospital and some of local nurses also get a chance of visiting the ideal set up for the short courses. This somehow has helped nurses to have insight of what our colleagues in the ideal world are doing; however not all had the privilege to obtain that exposure.

\section{Availability of policy on withdraw of treatment}

Study findings shows that, in Tanzania there is no protocol for withdraw of treatment for patients who are brain dead because as per our culture and beliefs every human has the right to treatments until God says stop. "I don't think it is right to withdraw treatments for anyone; it is only God who can do that. I would advocate allowing normal dearth to occur."

Nurses interviewed said that, there is no policy written down, they have neither heard nor seen it. "I have never come across any protocol as far as withdraw of treatment is concerned and I don't support anything like that". However they said, it is a logical thing to withdraw treatments because one cannot continue treatment of somebody who is brain dead and not hoping to achieve anything, all what is needed is to keep them comfortable but not to continue with aggressive treatments.

The above reaction from our respondents precisely shows that, people are strongly tied to their culture and beliefs and does not see the point of having such a protocol since they do not anticipate the practice to practice here in Tanzania knowing that religion and culture will not entertain such a thing.

\section{Equipment and specialized department (ICU)}

The findings shows that, the ICU still lack most of the items, consumable, medication and lifesaving equipment necessary for the provision of quality care to critically ill patients. Nurses said, besides ventilators there are many other things that are needed to make up complete unit which are now not available in the unit including ABG machine portable x-ray, Dialysis central lines, TPN (Feeds) etc. There is a need for suppliers' offices to order the above; shortage is of equipment is a big challenge, which make nurses run for greener pastures.

"Very inadequate to cater for the increasing number for terminal ill patients and I suggest more critical units which are well equipped with both human and non-human resources".

However in comparison to other ICU places set up in our country, the ICU set up in Muhimbili national hospital has tried so much, it just miss some equipment, nurses still need more and hoping that the management will keep ordering some more. 
Texila International Journal of Nursing

Volume 2, Issue 2, Dec 2016

\section{Supportive suspensions from the management}

The study findings shows that, so far there is no support from the management to prevent stress for the ICU nurses, because management does not appreciate the workload and stress that comes with work in the ICU or working with critically ill patients. There are no motivational incentives at all, people in management know nothing about ICU and it still a challenge to keep on telling them what is going on here until a person from management having a patient here and visiting to a patient, that is when they realize that, there is something that needs to be considered but for the time being nurses are working in a very stressful condition, sometimes nurses don't have food, and have to go out look for food, water and sugar. Resting sometimes is not there and leaders do not realize that ICU is a hectic place and consider it as a light duty.

Informants added that, management just consider the ICU nurses the same as other nurses, they treat ICU nurses same as other nurses overlooking the complexity and the demands that they face. But the reality is that, ICU nurses workload is very high and stressful both physically and psychologically hence they need to be taken care of to motivate them either financially, counseling, timeout and training. Seeing critically ill and dying patients daily is very stressful hence professional counseling is paramount otherwise burn out and fatigue will be eminent.

"Critical care workload is huge so even appreciation is vital, but it looks like the management does not differentiate us from the rest of nurses, I have a feeling that management does not have knowledge of what is going on in the unit. We need motivation in term of monetary, holidays, counseling or refresher courses."

Nurses suggested the following to be done by the management to motivate them which in turn will lead into effective provision of care to critically ill patients.

- Motivation packages should be provided like provision of food, refreshment, money, holidays and most of all counseling because it is not easy seeing people dying daily.

- Conducive environment to release stress such as music holidays or outings, money, lunch or tea since it is a close unit, more important counseling.

- There is a need for creating a conducive environment such as stress relieving modalities, outings, provision of food and refreshments, resting areas, soft music, monetary gains and even appreciation of work done more trainings are needed.

\section{Summary of findings, conclusion and recommendations}

\section{Introduction}

This chapter contains summary of findings, conclusions and recommendations drawn from the analysis. It is a highlight of issues that were revealed during the research. It also involves recommendations that can help management to overcome the challenges faced by nurses working in the ICU caring for critically ill patients.

\section{Summary of findings}

The aim of this study was to assess the perception and to examine the ethical dilemma faced by critical care providers in a tertiary teaching hospital in Tanzania when caring for terminal patients. The study was guided by four specific objectives including: first to assess how the critical care Nurses perceive and understands end of life issues encountered during caring for terminal patients, second to explore the ethical dilemmas faced by Nurses during the course of duty in caring for terminal ill client, third to find out the psychological impact of end of life issues to critical care providers working in the ICU and lastly to determine the challenges encountered by critical care providers working in the ICU.

The study was conducted from Muhimbili National Hospital (MNH) in Dar es Salaam, Tanzania. The population of the study was subgroup of nurses based on qualifications, expertise and experience, working from medical ICU, surgical ICU, cardiac ICU and CCU. All the nurses who met the above criteria were included in the study. The researcher applied both structure and unstructured interview to collect data. 
The study findings show that, majority of nurses understands and come across end of life issues more frequently in ICU and most of them find these issues very uncomfortable and have negative perception towards a number of such end of life issues for example they don't support the medical team to decide on withdrawing treatments, they would advocate for normal dearth to occur unless it is the patient himself who determined his/her fate by choosing what should be done as far as DNR is concerned or withdraw of the treatments, religiously and culturally nurses don't support it, they have a strong belief that God himself is the only one who can determine ones dearth despite the prognosis or brain death.

Second it was observed that, working in the ICU affects nurses both psychologically and physically, psychologically nurses have been affected especially when the patient dies regardless of the effort put to save the patient, the effect may progress to physical due to stress and fatigue which may end up prone to sickness such as frequent cold, flue and sometimes abdominal upset and the worst which is burnout which prevent nurses from giving the best care to their patients and families. It is not easy to see a patient in pain and suffering, knowing very well the prognosis is poor and the patient is not getting better and there is nothing one can do to stop the suffering.

Third, the report shows that nurses come across so many ethical dilemmas in the ICU which leave them with no best answer or solution. Most of the dilemmas are on truth telling; withdraw of treatment to vegetative patients and allocation of resources. In order to work out such dilemma, nurses tend to assess the situation and if possible involve colleagues since they work as a team. Together they analyze the situation and solve it the best way possible, so in the end of the day, no uncertainty on both parties.

Lastly nurses working in the ICUs experienced challenges when caring for ICU patients and their family members. The challenges included a lack of adequate ICU training and the absence of policies as well as inadequate equipment and drugs. Nurses were reportedly unprepared to provide sustained information to family members; abilities to rest; and to deal with specific religious and cultural practices. Nurses also experienced competing demands between caring for the ICU patients and providing support for these patients' family members.

\section{Conclusion}

The researcher concludes that, majority of nurses come across end of life issues more frequently in ICU and most of them find these issues very uncomfortable. Nurses have negative perception towards a number of End of life issues for example they don't support the medical team to decide on withdrawing treatments. The study further found that majority of nurses is psychologically and physically affected by end of life issues whereby most of them suffer from moral distress.

Ethical dilemmas encountered by nurses are mostly on truth telling; withdraw of treatment to vegetative patients and allocation of resources. Lastly the researcher found that, lack of adequate ICU training, lack of policies and protocol on withdraw of treatments and inadequate of equipment as the main challenges among nurses working in the ICU.

\section{Recommendations}

First and foremost, it has been clear in this research that more than half the nurses interviewed suffer a lot from moral distress due to the nature of their work and ethical dilemmas they encounter during their day to day practice, the current hospital system seems only to care more for the client who is a patient rather than the caregiver. I recommend the hospital management to also support their care givers, both psychologically and physical, through acknowledgement and recognition of the complex nature of critical care work as well as increased workload which is different from other departments.

Psychological support aspect the hospital must be ready to provide Counseling for the nursing staff whenever one shows signs of fatigue and burnt out through supportive supervision. A stressed nurse can never deliver quality care.

Also I recommend that mediate nurse supervisors in critical care nursing be personnel that have qualification and en sight of the clinical duties of the ICU nurse, it logic to supervise something that 
Texila International Journal of Nursing

Volume 2, Issue 2, Dec 2016

one knowledgeable as this will help the management to appreciate critical care work. The management should also come up with motivational programs and incentives such as recreation, meals, and cash or in kind.

Shortage of both human and non human resources, the management must ensure well staffing as per universal standards, ideally nurse patient ratio in ICU should be 1:1, the procurement department must ensure the availability of equipment and consumables which all the nurses cited as one of the biggest hindrance to quality care provision and contributes to their dilemmas and stress. The hospital should do enough capacity building to cater for the size of the hospital, ideally and universal standards says, at least $10 \%$ of the total hospital bed capacity should be able to be accommodated into the ICU, hence Muhimbili hospital with a bed capacity of 1500 beds, must have at least 150 ICU beds.

Training is another important aspect, to promote a better critical care practice; Critical care nursing is a specialty which calls for special formal training in order to attain the specialized skills and competency needed. Less than $2 \%$ of the current nursing staff in critical care are not formally trained, they are working through experience and on job training. Nurses should be send for formal training to acquire the competency skills that are required of a critical care nurse and be able to practice in such a demanding discipline.

Policy makers should also ensure there is a legislation on end of life issues, as of current Muhimbili hospital has no protocol as far as end of life issues are concerned, worse still the country has no policy on such issues, I urge the policy makers through the political will to pass a law which guide the health care providers when they are faced with such issues hence there would be uniformity in all the hospital nationally.

\section{Acknowledgement}

I acknowledge that this piece of work is my own, and dedicate it to my precious beloved Dad, who passed on just a few months before this study. MHSRIP!

Special thanks go to my supervisor Dr Edwin Lugazia of Muhimbili University of Allied health and technology (MUHAS) for tirelessly pushing and guiding me through this whole project.

To my kids, Prudence Tsitsi and Itayi Prince, not forgetting my little man grandson a.k.a. Mufaro, I salute you, all your encouragement made this possible.

Above all, am most grateful to God Almighty for making this dream come true, "Asanthesana, Ndinotenda, ngiyabonga." (Thank you).

\section{References}

[1]. American Bar Association Commission on Law and Aging. Trends in healthcare decisions legislation relevant to the PSDA. Available at: http://www.abanet.org/aging/psda.html. Accessed June 2, 2004.

[2]. Brysiewicz, P. 2008. The Lived experience of losing a loved one to a sudden death in KwaZulu-Natal, South Africa. Journal of Clinical Nursing, 17: 224-231.

[3]. Cantor MD, Braddock CH, 3rd, Derse AR, et al. Do-not-resuscitate orders and medical futility. Arch Intern Med. Dec 8-22 2003;

[4]. Carlson, K.K. 2009. Advanced critical care nursing. Canada: Saunders

[5]. Covinsky DI, Fuller JD, Yaffe K, et al. Communication and decision-making in seriously ill patients: findings of the SUPPORT project. J Am Geriatr Soc. 2000; 48(5 suppl):S187-193.

[6]. Curtis, J.R. 2001a. Communicating about end-of-life care with patients and families in the intensive care unit. Critical Care medicine (online), 20(3): 363-380. Available WWW: http://www.ncbi.nlm.nih.gov [Accessed on the 10 July 2011)

[7]. Dracup, K. and Bryan-Brown, C.W. 2005. Dying in the Intensive Unit. American Journal of Critical Care, 14(1): 456-458.

[8]. Elliot, R.M. and McKinley, S. 2008. Implications for Australian practice of North American guidelines for the support of the family in patient centered intensive care. Collegian (online), 15(1): 11-7. Available WWW: http://www.ncbi.nlm.gov [Accessed on the 7 October 2011]

[9]. Eyetsemitan, F. 2002. Cultural interpretation of dying and death in a non-Western society: The case of Nigeria. Reading in Psychology and Culture. 14 (1): 1-6. 
[10]. Fouche, N.A. 2006a. The Last hours of living in the ICU-priorities of care for critical care nurses. The Southern African Journal of Critical Care (online), 22(2): 65-67. Available WWW: http:// www.sajcc.org.za [Accessed on the 28 September2011]

[11]. Fridh, I., Forsberg, A and Bergbom. I. 2007. Family presence and environmental factors at time of a patient's death in ICU. Acta Anesthesia Scand, 51: 395-401.

[12]. Glaser, B.G and Strauss, A.L. 2004. Awareness of dying. Aldine: Sociology Press

[13]. Gilllick M. Advance care planning. N Engl J Med. 2004; 350(1):7-8

[14]. Hardwig, J. 2000. Spiritual issues at the end-of-life: Hastings Centre Report 30(2): 28-30

[15]. Hudak, MC. Morton, GP. Fontaine, KD and Gallo, MB. 2008. Critical care nursing: A holistic approach. 8th ed. Philadelphia: Lippincott Williams \& Wilkins

[16]. Klein, S. and Alexander, D.A. 2003. Good grief: A medical challenge. Trauma.

[17]. 5(1): 261-271

[18]. Langley, G. and Schmollgruber, S. 2006. End-of-life care in intensive care units. Southern African Journal of Critical care (online), 22(2)58-65. Available WWW: http:// www.sajcc.org.za [Accessed on the 28 September 2011]

[19]. Lee, B.S. 1996. Registered nurses' experiences of caring for a dying family member. Masters Dissertation, BSN, University of Victoria.

[20]. Lo B. Resolving Ethical Dilemmas: A Guide for Clinicians. 2nd ed. Philadelphia, PA: Lippincott Williams \& Wilkins; 2000.

[21]. Lo B. Confusing ethical distinctions. In: Resolving Ethical Dilemmas: A Guide for Clinicians. Philadelphia, PA: Lippincott Williams \& Wilkins; 2000:119-128.

[22]. Lo B. Tube and intravenous feedings. In: Resolving Ethical Dilemmas: A Guide for Clinicians. Philadelphia, PA: Lippincott Williams \& Wilkins; 2000:145-150.

[23]. Lynn J. Perspectives on care at the close of life. Serving patients who may die soon and their families: the role of hospice and other services. JAMA. Feb 21 2001; 285(7):925-932.

[24]. Mani, R.K. 2003. Limitation of life support in an ICU: end of care ethical issues relating to end-of-life care. Indian Journal of Critical care Medicine, 7: 112-117

[25]. Martin N Marshall. Sampling for qualitative research. Vol 13, No 6. Family practice Oxford University press 1996.

[26]. Marco CA, Schears RM. Societal opinions regarding CPR. Am J Emerg Med. 2002; 20:207-211?

[27]. Michell, L. 2010. Crash and burn. Southern African Journal of Critical Care 26(2): 34.

[28]. Morton, G.P. Fontaine, K.D. Hudak, M.C and Gallo and M.B. 2008. Critical Care Nursing: A Holistic Approach. 8th ed. Philadelphia: Lippincott Williams \& Wilkins

[29]. Navaran F. From "What Is the Difference Between Ethics, Morals and Values?" Available at: http://www.ethics.org/ask_e4.html. Accessed December 28, 2004

[30]. Niederriter, J.E. 2009. Student nurses' perception of death and dying. Doctor of Philosophy in Urban Education, Cleveland State University.

[31]. Prompahakul, B.S.N., Nilmanat, K and Kongsuwan, W. 2011. Factors relating to nurses' caring behaviors for dying patients. Nurse Media Journal of Nursing 1(1): 15-27.

[32]. Reisfield G, Wilson G. Advance car planning redux: it’s time to talk. Am J Hosp Palliat Med. 2004; 21:164-166.

[33]. Salman, K. and Zoucha, R. 2010. Considering faith within culture when caring for the terminally ill Muslim patient and family. Journal of Hospice and Palliative Nursing. 12 (3): 156-163

[34]. Searle, C., Human, S. and Mogotlane, S.M. 2009. Professional practice: A South African nursing perspective.5th ed. Johannesburg: Heinemann.

[35]. Searle, C. 2000. Professional Practice: A South African nursing perspective.4th ed. Johannesburg: Heinemann

[36]. Teno JM, Hakim RB, Knaus WA, et al. Preferences for cardiopulmonary resuscitation: physician-patient agreement and hospital resource use. The SUPPORT Investigators. J Gen Intern Med. Apr 1995;

[37]. Urden, L.D., Stacy, K.M and Lough, M.E. 2010. Critical care nursing: Diagnosis and management. 6th ed. St Louis: Mosby 
Texila International Journal of Nursing

Volume 2, Issue 2, Dec 2016

[38]. Vincent, J.L. 2006. End-of-life practice in Belgium and new euthanasia law. Intensive Care Medicine, 32: 1908-1911.

[39]. Wingate, S. and Wiegand, D.L. 2008. End-of-life care in the critical care unit for patients with heart failure. Critical Care Nurse (online), 28(2): 84-95. Available WWW: http://www.cconline.org [Accessed on the 4 October 2011]

[40]. Zomorodi, M. and Lyn, M.R. 2010. Critical care nurses’ values and behaviors with end-of-life. 